\title{
Original article \\ The vagina has reducing environment sufficient for activation of Trichomonas vaginalis cysteine proteinases
}

\author{
J F Alderete, Daniele Provenzano
}

\begin{abstract}
Background: Trichomonas vaginalis, a worldwide distributed sexually transmitted protozoan, is remarkable for synthesis of numerous, distinct cysteine proteinases, the significance of which is evidenced by the presence in vivo of soluble proteinases in secretions and antiproteinase antibody in serum of patients with trichomonosis. These proteinases purportedly play a role in host parasitism and immune evasion.

Objective: It is known that for cysteine proteinases to be functional, they must be activated by disulphide reducing reagents. Whether or not the host vaginal environment has the reducing environment essential for activation of the trichomonad cysteine proteinases is unknown. Our goal, therefore, was to determine whether or not vaginal secretions had sufficient reducing power to activate the trichomonad proteinases.

Methods: 48 vaginal washes (VWs) from patients were assayed for reducing equivalents and a score in dithiothreitol (DTT) reducing equivalents was assigned to each VW. Activation of trichomonad cysteine proteinases was then tested under the range of reducing equivalents detected from VWs. The possible protective effect of hydrogen peroxide, an oxidising agent produced by some Lactobacillus species, on proteinase activity was also determined.

Results: Nine of $48 \mathrm{VWs}(18.7 \%)$ possessed $\leqslant 10 \mu \mathrm{M}$ DTT reducing equivalents, four VWs (8.3\%) had from $20 \mu \mathrm{M}$ DTT to $40 \mu \mathrm{M}$ DTT reducing equivalents, and most (50\%) were between $10 \mu \mathrm{M}$ to $15 \mu \mathrm{M}$. Overall, the range in VWs was from $\sim 10 \mu \mathrm{M}$ to $40 \mu \mathrm{M}$ reducing equivalents. Importantly, data suggest differential proteinase activation over this in vivo range of reducing level. Only two $T$ vaginalis cysteine proteinase activities were stimulated at $2 \cdot 5 \mu \mathrm{M}$ DTT in contrast with all proteinase activities present at $40 \mu \mathrm{M}$ DTT, albeit quantitatively diminished compared with the activity at $1 \mathrm{mM}$ DTT, the concentration routinely used in vitro. Finally, hydrogen peroxide reversibly neutralised all trichomonad proteinases.

Conclusions: These results show that the vagina of women has a reducing environment adequate for activation of trichomonad proteinases. The data underscore that the host environment plays a role in the host-parasite interrelation. Finally, hypotheses can now be formulated to help explain resistance and susceptibility to infection commonly reported among women and between men and women with trichomonosis.

(Genitourin Med 1997;73:291-296)
\end{abstract}

Keywords: Trichomonas vaginalis; vagina; cysteine proteinase

\section{Introduction}

The host-Trichomonas vaginalis interrelation is exceedingly complex. Infection with this sexually transmitted parasite often leads to unpredictable outcomes in symptomatology ${ }^{1-3}$ that range from a carrier state to severe foul smelling discharge, irritation, and discomfort associated with inflammation and tissue cytopathology. ${ }^{1-3}$ It is now appreciated that patients with trichomonosis (vaginitis) are at higher risk for HIV seroconversion, ${ }^{45}$ for adverse outcomes during pregnancy, ${ }^{6-8}$ and, possibly, for cervical cancer..$^{910}$ The identity of factors, either of host or parasite origin, responsible for the variations in host responses to $T$ vaginalis infection remains unknown.

Recently, trichomonad cysteine proteinases have been recognised as virulence factors. ${ }^{11-16}$ The parasites generate numerous cysteine proteinases, ${ }^{17-19}$ many of which are secreted during normal cultivation in complex medium. The in vivo relevance of the proteinases is affirmed by the detection of proteinases and antiproteinase antibody in vaginal secretions and in sera of women with trichomonosis, respectively, but not women who are uninfected or who have other sexually transmitted diseases. ${ }^{111620}$ These enzymes probably contribute to numerous virulence properties of $T$ vaginalis organisms, such as acquisition of nutrients, ${ }^{21}$ subversion of host immune responses, ${ }^{13} 16$ and, through unknown mechanisms, parasite cytoadherence. ${ }^{12}$

Substrate degradation by cysteine proteinases requires breakage of disulphide bonds, as occurs under reducing conditions. ${ }^{17-1922}$ In this study, we have attempted to address the question of whether the vaginal environment of patients has sufficient reducing levels to activate trichomonad cysteine proteinases. This is important because all studies performed to date have activated the cysteine proteinases by addition of reducing reagents such as DTT. Equally important, it has been hypothesised that the host environment may play a role in regulating, both quali- 
tatively and quantitatively, the numerous trichomonad proteinases thereby contributing to a chronic, non-self limiting infection and minimal manifestation of tissue cytopathology.

In this report we show that vaginal secretions obtained from patients with trichomonosis do indeed possess an environment adequate for activation of the cysteine proteinases. It is noteworthy that the reducing levels produced differential activation or quantitatively distinct amounts of proteinases. These data reinforce the idea that variations in reducing levels in vaginal secretions play a role in infection by $T$ vaginalis and possibly in disease outcome. The observations made in this report point to future questions that must be answered to fully understand this complex host-parasite interaction and to meaningfully develop future infection and/or disease intervention strategies.

\section{Materials and methods}

CULTURES

$T$ vaginalis isolates IR78, NYH 286, T048, and T068-II were grown at $37^{\circ} \mathrm{C}$ in trypticase yeast extract maltose (TYM) supplemented with $10 \%$ heat inactivated horse serum (HIHS), as described before. ${ }^{2324}$ To optimally visualise for trichomonad cysteine proteinase activities in substrate gels, as described below, isolate T068-II was also grown under low iron conditions in the presence of $30 \mu \mathrm{M} 2,2-$ dipyridyl $(2,2-\mathrm{DP}) .{ }^{25} 26$ Organisms $\left(2 \times 10^{7}\right)$ were collected at mid to late logarithmic phase of growth ${ }^{23}$ by centrifugation at $500 \times g$ for 5 minutes after washing three times in ice cold phosphate buffered saline (PBS). Cells were either used immediately or frozen at $-70^{\circ} \mathrm{C}$ until needed. ${ }^{16}$ Identical proteinase patterns were obtained with lysates derived from either fresh or frozen parasites, as before. ${ }^{16}$

DETERMINATION OF REDUCING LEVEL IN HUMAN VAGINAL WASHES

To maximise detection of reducing ability, vaginal washes (VWs) were obtained from the source previously reported by us and processed as described before. ${ }^{1127}$ Briefly, a volume of $5 \mathrm{ml}$ of a VW was obtained in sterile PBS by rinsing the vaginal wall numerous times. To this VW a large swab, which was utilised to scrape the vaginal wall, was then immersed in the VW from the same patient. VW was then clarified as previously described by centrifugation at $500 \times g$ to remove large cellular debris followed by centrifugation at $17500 \times g$ to remove additional insoluble debris. The VWs were kept stored at $-70^{\circ} \mathrm{C}$ until utilised. Upon thawing and just before use, VWs were filter sterilised through $0.22 \mu \mathrm{m}$ acrodisc filters (Gelman Sciences, Ann Arbor, MI, USA).

The reducing potential of patient VWs was determined spectrophotometrically at $412 \mathrm{~nm}$ by comparing readings with a standard curve derived from a buffer comprised of known concentrations of DTT in Ellman's reagent $\left(5,5^{\prime}\right.$-dithio-bis (2-nitrobenzoic acid; DTNB $\left.)\right)^{28}$ (100 mM TRIS-HCl, pH 8.0, $100 \mathrm{mM}$
EDTA, $10 \mathrm{mM}$ DTNB, $50 \mathrm{mM} \mathrm{NaPO}$, pH $7 \cdot 0,10 \%$ sodium dodecylsulphate (SDS)). Reducing values of VWs diluted in DTNB were obtained from the average of triplicate determinations, and values were expressed as $\mu \mathrm{M}$ DTT equivalents. Reducing equivalents $\leqslant 10 \mu \mathrm{M}$ DTT were below the detection limit of DTNB and, therefore, represented as $\leqslant 10$.

DETECTION OF TRICHOMONAD CYSTEINE

PROTEINASE ACTIVITY

Protocols for preparation of parasite lysates? and for substrate gel electrophoresis have been $\overrightarrow{\overline{\hat{S}}}$ extensively described by us and others. ${ }^{16-1922} \vec{O}$ Pelleted parasites were solubilised in $200 \mu \mathrm{l}$ electrophoresis dissolving buffer ${ }^{29}$ (125 mM $\frac{\bar{\rho}}{\neg}$ TRIS-HCl, pH $6 \cdot 8,4 \%$ SDS, $20 \%$ glycerol, $\stackrel{ه}{\complement}$ and $10 \%$ bromophenol blue) in the absence of $\%$ $\beta$ mercaptoethanol. ${ }^{16}$ Samples $(1 \mu \mathrm{l})$ were elec- $\vec{\circ}$ trophoresed at $100 \mathrm{~V}$ in a minigel apparatus (BioRad Laboratories, Hercules, CA, USA) $\vec{\omega}$ on $7 \%$ acrylamide copolymerised with $1.5 \mathrm{mg}$ gelatin, as before. ${ }^{16-1822}$

After SDS-PAGE, trichomonad proteinases were activated in proteinase activation buffer $\underset{\mathscr{c}}{\mathrm{g}}$ (PAB) (100 mM sodium acetate and $2.5 \%$ Triton-X $100, \mathrm{pH} 5 \cdot 5)$ prewarmed to $37^{\circ} \mathrm{C}$ 은 containing varying concentrations of DTT. In this case, preparative slab gels were cut so that identical amounts of trichomonad protein $\frac{c}{0}$ were incubated separately in PAB containing specific amounts of DTT. Negative controls $\bullet$ were incubated in PAB without any addition. PAB with hydrogen peroxide $(10 \mu \mathrm{M})$ was also tested to determine the effect on proteinase activity. ${ }^{3031}$ Activation of proteinases was performed by placing gels on a shaker for $2 \frac{\circ}{\Phi}$ hours at $37^{\circ} \mathrm{C}$ with one change of buffer, and proteinase activity was seen after staining in Coomassie brilliant blue overnight followed by destaining for 2 hours in a solution of $7 \%$ acetic acid and 5\% methanol.

DENSITOMETRIC ANALYSIS OF SUBSTRATE GELS FOR DETERMINATION OF RELATIVE PROTEINASE ACTIVITY

All gels were digitalised with a flatbed scanner, 은 and pixel intensity of each clear area repre- $D$ senting a proteinase degradation band was quantified and graphed with the NIH Image $\stackrel{\sim}{\circ}$ software version $5 \cdot 17$. Percentage degradation for each band was calculated as follows: $100 \div$ ((no pixels scanned from gels activated at $1 \mathrm{mM}$ DTT) $\times($ no pixels scanned from gels activated at $<1 \mathrm{mM}$ DTT)) .

\section{Results}

APPROXIMATION OF REDUCING LEVELS IN VWS We did not know whether it would be possible to measure any reducing ability in VWs obtained and processed as described in Materials and 8 methods. We further appreciated that any value of reducing level detected may not reflect that found at a specific site in the vagina parasitised by $T$ vaginalis. None the less, the question regarding whether the vagina possessed an environment sufficient to activate trichomonad proteinases was important to attempt determination of in vivo reducing levels. 


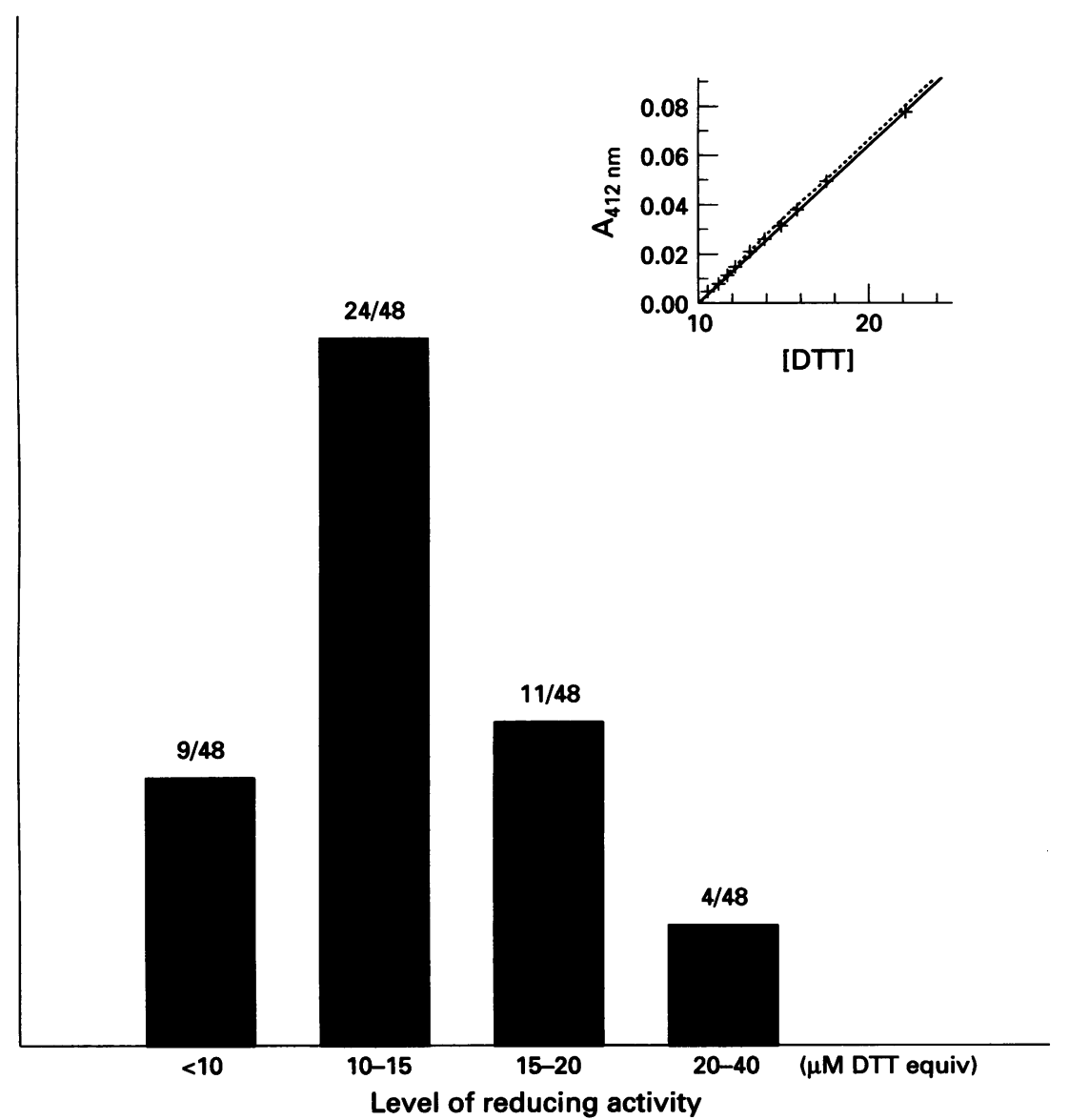

Figure 1 Distribution of reducing levels among the vaginal washes of patients with trichomonosis. On top of each bar are the numbers of samples positive for each range. $A$ total of 48 vaginal washes was examined. The inset shows the standard curve using different concentrations of dithiothreitol (DTT), and a representative set of VWs analysed is represented by the plus signs. The protocol was as described in Materials and methods. Absorbance was monitored at $412 \mathrm{~nm}$ wavelength.

Figure 1 presents the results from the evaluation of $48 \mathrm{VWs}$ and extrapolation of DTT equivalent levels from a standard curve using $\mu \mathrm{M}$ amounts of DTT (inset). The majority of the VWs (50\%) had DTT reducing equivalents between $10 \mu \mathrm{M}$ and $15 \mu \mathrm{M}$. Nine of 48 (18.7\%) VWs assayed had $\leqslant 10 \mu \mathrm{M}$ DTT equivalents. In contrast, 11 of $48(23.0 \%)$ had



Figure 2 Representative cysteine proteinase patterns of $T$ vaginalis by substrate gel electrophoresis with gelatin copolymerised with acrylamide using varying amounts of dithiothreitol (DTT) (lanes 1-8) in the activation buffer (Materials and methods). None refers to the control without any DTT (lane 9). Lane 10 shows the effect of addition of hydrogen peroxide $\left(\mathrm{H}_{2} \mathrm{O}_{2}\right)$ as described in the text. The letters on the left refer to proteinase band designations for comparative purposes as shown in figure 3 and in the table. Similar results were obtained with numerous isolates examined, including those listed in Materials and methods.

between $15 \mu \mathrm{M}$ and $20 \mu \mathrm{M}$ and four VWs $(8.3 \%)$ had $20 \mu M$ to $40 \mu M$ DTT equivalents. This range ( $\leqslant 10 \mu \mathrm{M}$ to $40 \mu \mathrm{M})$ was far lower than the $1 \mathrm{mM}$ amount of DTT normally employed to examine the trichomonad cysteine proteinases in vitro. ${ }^{181922}$ None the less, these data show that VWs from patients do indeed possess reducing ability.

ARE VW REDUCING LEVELS SUFFICIENT TO ACTIVATE TRICHOMONAD CYSTEINE PROTEINASES?

We next wanted to determine whether cysteine proteinases were detected when activation was performed using concentrations of DTT within the range found in the VWs. In addition, because the manner in which the VWs were obtained and processed could yield lower values than those of undiluted vaginal secretions, we evaluated reducing levels from $10 \mu \mathrm{M}$ up to $1 \mathrm{mM}$ for comparative purposes.

Figure 2 illustrates proteinase profiles of lysates corresponding to $1 \times 10^{5} T$ vaginalis isolate T068-II organisms where gels were treated with decreasing amounts of DTT (lanes 1-9). Lysate of this parasite density was used because, by substrate gel electrophoresis, individual proteinase band activities were best resolved for accurate visualisation and comparisons. ${ }^{16}$ This amount of lysate also gives reproducible total proteinase patterns among different $T$ vaginalis isolates that were examined identically as presented here and for which similar results were obtained. Equally important, the number of parasites is relevant to that found in vivo. ${ }^{32}$ Finally, that the degrading activities being analysed are cysteine proteinases has been confirmed using specific inhibitors. ${ }^{16-1822}$

Amounts of DTT above $100 \mu \mathrm{M}$ (lane 2) gave patterns similar to $1 \mathrm{mM}$ (lane 1 ). By contrast, beginning at $100 \mu \mathrm{M}$ (lane 2), there was a decrease in total proteinase activity, especially in the lower $M_{\mathrm{T}} \mathrm{s}$ (lane 1 versus lanes 3 ). Concentrations of DTT as low as $2.5 \mu \mathrm{M}$ still gave detectable proteinase activity above background, as seen in lane 9 (greater than the negative control without addition of any reducing agent).

We had noticed that any basal level of proteinase activity observed in the negative controls could be readily neutralised by addition of $10 \mu \mathrm{M}$ hydrogen peroxide in the activation buffer (lane 10). We, therefore, thought it would be important to determine whether hydrogen peroxide neutralised the activity of DTT pretreated cysteine proteinases. Hydrogen peroxide is known to reverse the effect of DTT, ${ }^{3031}$ and, as recently hypothesised, hydrogen peroxide producing Lactobacillus might therefore afford protection from $T$ vaginalis infection. ${ }^{33-35}$

Identical experiments as above were performed in substrate gels that were activated in $40 \mu \mathrm{M}$ DTT, the minimum concentration found in VWs that gave good activation of the trichomonad cysteine proteinases (fig 2), except that activation buffer incorporated varying amounts of $\mathrm{H}_{2} \mathrm{O}_{2}$. At $200 \mu \mathrm{M} \mathrm{H} \mathrm{H}_{2}$, no activation of proteinases was detectable, 
Figure 3 Densitometric scanning of the proteinase bands obtained after activation at the

designated dithiothreitol (DTT) concentrations. The surface under each peak was analysed as described in Materials and methods for quantitative presentation in the table. The dotted line was used to align each peak.



showing that hydrogen peroxide neutralised the action of the reducing environment needed for the cysteine proteinases.

To better quantitate the proteinase activities, we performed densitometric scanning (fig 3) and evaluated each prominent gel band region labelled $A$ to $G$ (fig 3 and table).
Proteinase bands $F$ and $G$ were greatly decreased by $100 \mu \mathrm{M}$ DTT (table) and were reduced in activity by $90 \%$ at $50 \mu \mathrm{M}$ DTT. Prominent substrate degradation by proteinase bands $\mathrm{D}$ and $\mathrm{E}$ were also strongly affected by a decline in DTT concentration; a $\sim 50 \%$ reduction in proteolysis occurred by $100 \mu \mathrm{M}$ DTT, and $\sim 80 \%$ activity was diminished by $50 \mu \mathrm{M}$ DTT. Band C was decreased $50 \%$ in activity by $50 \mu \mathrm{M}$ DTT, while band regions $A$ and $B$ approached a $50 \%$ reduction 3 in activity by $20 \mu \mathrm{M}$ DTT. Therefore, when $\stackrel{\circ}{\circ}$ evaluating the level of DTT at which a $50 \% \vec{\Rightarrow}$ reduction in activity is detected, differences $\stackrel{5}{9}$ are apparent among the proteinase band? regions.

\section{Discussion}

The interrelation between the host and Trichomonas vaginalis is exceedingly complex, $\overrightarrow{\vec{\omega}}$ and some of the many factors that contribute to such complexity include the multiple cys- $\stackrel{\text { ? }}{=}$ teine proteinases that potentially contribute to $\omega$ host cytopathology. We have previously hypothesised that the in vivo synthesis of the $c$ proteinases must somehow be under the con- 0 trol of environmental cues so as to modulate the number and amount of proteinases needed $\supset$ at any particular moment of infection. In thisco scenario only that quantity of proteinases nec- $\stackrel{\infty}{\stackrel{\rho}{\rho}}$ essary for parasite survival and which provoke $\vec{c}$ minimal host damage would be both present and active. Recent evidence shows the induction by iron of proteinases with specific functions, such as degradation of complement $\mathrm{C} 3$ to avoid lysis through the alternative pathway. ${ }^{13}$ In addition to regulatory paths that con- $\triangle$ trol the synthesis of the trichomonad $\vec{\overrightarrow{ }}$ proteinases, we now present the possibility that host reducing levels may also regulate the activity of the proteinases. This would provide another mechanism of control over the many proteinases produced by $T$ vaginalis.

Of importance was the finding that VWs indeed possess reducing ability. To our knowl- $\stackrel{\circ}{\Omega}$ edge, this may be the first time that the question regarding whether, during infection, in 5 vivo conditions are suitable to activate the tri- $-\frac{D}{O}$ chomonad proteinases has been addressed. In this report, we show that VWs of patients with trichomonosis have reducing ability for activa- $N$ tion of the cysteine proteinases, albeit the levels detected by us were at 20 -fold lower concen-

The effect of reducing level on relative activity of $T$ vaginalis cysteine proteinases

Relative activity was calculated as described in Materials and methods.

†Band designations were from the individual areas of proteinase activity as presented in figures 2 and 3 .

$¥$ Minus and plus signs refer to the levels of reducing ability in DTT equivalents as determined from the evaluations of vaginal washes as shown in figure 1 and described in Materials and methods. 
trations than those used in vitro. ${ }^{16-1922}$ It is conceivable that the lower amounts of reducing levels represent a dilution resulting from obtaining and/or processing the VW. Regardless, it is clear that lower amounts of reducing ability produce qualitatively and quantitatively decreased activity of parasite proteinases when compared with $1 \mathrm{mM}$ DTT. Finally, it might have been predicted that all the proteinase activities would decrease equally at a particular reducing level. That this was not the case reaffirms the distinctness among the numerous cysteine proteinases, as suggested earlier. ${ }^{17}$

It is essential that we begin to understand the host factors and parameters within the site of infection that contribute to individual susceptibility and resistance to infection. The hypothesis that women with higher vaginal reducing ability may be more susceptible to parasitism and cytopathology seems reasonable, especially given the relation between qualitative and quantitative proteinase activities and differential reducing levels. This idea is plausible since proteinase activity is necessary for survival of $T$ vaginalis through nutrient acquisition, ${ }^{21}$ cytoadherence, ${ }^{12}$ and immune evasion. ${ }^{1316}$

If the hypothesis presented above is correct, then relative resistance by men to infection by $T$ vaginalis may be related to the presence of oxidising reagents that prevent activation of the trichomonad cysteine proteinases essential for survival. In fact, reactive oxygen species produced by human spermatozoa have been described, ${ }^{36}{ }^{37}$ and this oxidative environment would not allow for host parasitism and establishment of a non-self limiting infection.

We showed the action of hydrogen peroxide in neutralising the reducing ability of DTT, which is a prerequisite for activation of the cysteine proteinases. Our results reinforce the notion that hydrogen peroxide producing Lactobacillus may confer some measure of defence against $T$ vaginalis. This is only possible, however, if sufficient amounts of hydrogen peroxide are produced at and bathe the site of infection. The idea to test for neutralisation of trichomonad proteinases resulted from the known reversible effect by hydrogen peroxide on systems requiring reducing environments. ${ }^{303138}$ Furthermore, the idea may have merit because of the reports that the host may be protected from other sexually transmitted diseases by hydrogen peroxide producing Lactobacillus. ${ }^{33-35}$ Although reversible, demonstration of hydrogen peroxide neutralisation of the activation power of DTT on trichomonad proteinases suggests that an approach for intervention of $T$ vaginalis infection and cytopathology may result from generation of irreversible proteinase inhibitors or, alternatively, upon inhibition of the ability of the parasite to alter the normal protective flora of patients.

Based on the hypothesis proposed above, numerous questions require attention. (1) Does the level of vaginal reducing ability vary during menses, and, if so, are certain times during the cycle more favourable to proteinase activation, which then promotes host parasitism? (2) Does the relative reducing level in the vagina vary among different ethnic groups, thus explaining higher rates of trichomonosis among African American women?6 (3) Does behaviour that predisposes women to $T$ vaginalis infection, such as smoking, ${ }^{6}$ alter the vaginal reducing environment? (4) Is there a relation between the level of reducing ability, parasite growth and multiplication in vivo, and symptomatology? (5) Does the relative absence of symptomatology and the self limiting nature of infection among males correlate with absence of reducing ability at those sites? Our results provide a foundation from which testable hypotheses can be formulated and that may, ultimately, help us understand those important host factors pertinent to infection by this sexually transmitted agent.

This study was supported by Public Health Service grant AI 31498 from the National Institute of Allergy and Infectious Diseases. We gratefully acknowledge Ed Newton and Jeana Piper of the Department of Obstetrics and Gynecology of the Piper of the Department of Obstetrics and Gynecology of the UTHSCSA for their contribution of fresh clinical isolates and vaginal washes through the Collaborative STD Research
Center. We thank Alan Barbour, Paul Horowitz, and Neal Center. We thank Alan Barbour, Paul Horowitz, and Neal
Robinson and their staff for allowing us to use their densitometry equipment and software. We are grateful to Jean Engbring for her critical review of our manuscript and for her suggestion throughout these studies. Lastly, we thank Tonya Williams for her expert secretarial assistance in the preparation of this manuscript.

1 Krieger IN, Wolner-Hanssen P, Stevens C, Holmes KK Characteristics of Trichomonas vaginalis isolates from women with and without colpitis macularis. $\mathcal{F}$ Infect Dis 1990;161:307-11.

2 Krieger JN. Trichomoniasis in men: old issues and new data. Sex Transm Dis 1995;22:83-96.

3 Müller, M. Trichomonas vaginalis and other sexually transmitted protozoan infections. In: Holmes $\mathrm{KK}$, Mardh $\mathrm{P}$ eds. International perspectives of neglected sexually transmitted disease. New York: Hemisphere Publishing, 1983:113-24.

4 Laga M, Manoka A, Kivuvu M, Malele B, Tuliza M, Nzila $\mathrm{N}$, et al. Non-ulcerative sexually transmitted diseases as risk factors for HIV-1 transmission in women: results risk factors for HIV-1 transmission in wom
from a cohort study. AIDS 1993;7:95-102.

5 Nzila N, Laga M, Thiam MA, Mayimona K, Edidi B, Van Dyck E, et al. HIV and other sexually transmitted diseases among female prostitutes in Kinshasa. AIDS $1991 ; 5: 715-21$

6 Cotch, MF, Patorek II JG, Nugent RP, Yerg DE, Martin DH, Eschenbach DA. Demographic and behavioral predictors of Trichomonas vaginalis infection among pregnan women. Obstet Gynecol 1991;78:1087-92.

7 Hardy PH, Hardy JB, Nell EE, Graham DA, Spence MR, Rosenbaum RC. Prevalence of six sexually transmitted disease agents among pregnant inner-city adolescents and pregnancy outcome. Lancet 1984;ii:333-7.

8 Minkoff H, Gruenebaum AN, Schwartz RH, Feldman J, Cummings $M$, Cromleholme $\mathbb{W}$, et al. Risk factors for prematurity and premature rupture of membranes: prospective study of the vaginal flora in pregnancy. $A m \mathcal{F}$ prospective study of the vagin

9 Bertini B, Hornstein M. The epidemiology of trichomonosis and the role of this infection in the development of carcinoma of the cervix. Acta Cytol 1970;14:325-32.

10 Zhang Z, Begg CB. Is Trichomonas vaginalis a cause of cervical neoplasma? Results from a combined analysis of 24 studies. Int ₹ Epidemiol 1994;23:682-90.

11 Alderete JF, Newton E, Dennis C, Neale KA. Antibody in sera of patients infected with Trichomonas vaginalis is to
trichomonad proteinases. Genitourin Med 1991;67:331-4.

12 Arroyo R, Alderete JF. Trichomonas vaginalis proteinase activity is necessary for parasite adherence to epithelial activity is necessary for parasite ad
cells. Infect Immun 1989;57:2991-7.

13 Alderete JF, Provenzano D, Lehker MW. Trichomonas vaginalis resistance to complement is mediated by ironinducible cysteine proteinase. Microbial Path 1995;19: 93-103.

14 Bozner P, Gambosova A, Valent M, Demes P, Alderete JF. Proteinases of Trichomonas vaginalis: antibody response in patients with urogenital trichomoniasis. Parasitol 1992, 105:387-91.

15 Garber GE, Lemchuck-Favel LT. Characterization of extracellular protease of Trichomonas vaginalis. Can $\mathcal{F}$ Microbiol 1989;35:903-9.

16 Provenzano D, Alderete JF. Analysis of human immunoglobulin-degrading cysteine proteinases of Trichomonas vaginalis. Infect Immun 1995;63:3388-95.

17 Neale KA, Alderete JF. Analysis of the proteinases of representative Trichomonas vaginalis isolates. Infect Immun
1990;58:157-62.

18 Coombs GH, North MJ. An analysis of the proteinases of 
Trichomonas vaginalis by polyacrylamide gel electrophoresis. Parasitol 1983;86:1-6.

19 North MJ, Robertson CD, Coombs GH. The specificity of trichomonad cysteine proteinases analyzed using fluorogenic substrates and specific inhibitors. Mol Biochem Parasitol 1990;39:183-94.

20 Alderete JF, Newton E, Dennis C, Neale KA. The vagina of women infected with Trichomonas vaginalis has numerous proteinases and antibody to trichomonad proous proteinases and antibody to trich

21 Dailey DC, Chang TH, Alderete JF. Characterization of Trichomonas vaginalis haemolysis. Parasitol 1990;101: 171-5.

22 Lockwood BC, North MJ, Scott KI, Bremner AF, Coombs GH. The use of a highly sensitive electrophoretic method to compare the proteinases of trichomonads. Molec Biochem Parasitol 1987;24:89-95.

23 Peterson KM, Alderete JF. Host plasma proteins on the surface of pathogenic Trichomonas vaginalis. Infect Immun 1982;37:755-62.

24 Diamond LS. The establishment of various trichomonads of animal and man in axenic cultures. $f$ Parasitol 1957 ; of animal and

25 Lehker MW, Alderete JF. Iron regulates growth of Trichomonas vaginalis and the expression of immunogenic trichomonad proteins. Molec Microbiol 1992;6:123-32.

26 Lehker MW, Arroyo R, Alderete JF. The regulation by iron of the synthesis of adhesins and cytoadherence levels in the protozoan Trichomonas vaginalis. 7 Exp Med 1992; 174:311-8.

27 Alderete JF. Enzyme-linked immunosorbent assay for detecting antibody to Trichomonas vaginalis: use of whole cells and aqueous extract as antigen. Br $\mathcal{F}$ Vener Dis 1984; 60:164-70.

28 Habeeb AFSA. Reaction of protein sulfhydryl groups with
Ellman's reagent. Methods Enzymol 1972;25:457-64.

29 Laemmli UK. Cleavage of structural proteins during the assembly of the head of bacteriophage T4. Nature 1970; 227:680-5.

30 Wright $M$, Drummond GI. Inactivation of the $\beta$-adrenergic receptor in skeletal muscle by dithiols. Biochem Pharmacol 1983;32:509-15.

31 Neumann NP. Oxidation with hydrogen peroxide. Methods Enzymol 1967;11:485-7.

32 Demes $P$, Gombosova A Valent $M$, Janoska A, Fabusova H, Petrenko M. Differential susceptibility of fresh Trichomonas vaginalis isolates to complement in menstrual blood and cervical mucus. Genitourin Med 1988; 64:176-9.

33 Hillier SL, Krohn MA, Klebanoff SJ, Eschenbach DA. The relationship of hydrogen peroxide-producing lactobacilli to bacterial vaginosis and genital microflora in pregnant women. Obstet Gynecol 1992;79:369-73.

34 Hughes VL, Hillier SL. Microbiologic characteristics of Lactobacillus products used for colonization of the vagina. Obstet Gynecol 1990;75:244-8.

35 Klebanoff SI, Hillier SL, Eschenbach DA, Waltersdorph AM. Control of the microbial flora of the vagina by $\bar{\omega}$ AM. Control of the microbial flora of the vagina by $\mathrm{H}_{2} \mathrm{O}_{2}-\mathrm{g}$

36 Aitken RJ, Buckingham D. Enhanced detection of reactive oxygen species produced by human spermatozoa with 7dimethyl amino-naphthalin-1,2-dicarbonic acid hydrazide. Intl $\mathcal{F}$ Androl 1992;15:211-9.

37 Rufas O, Fisch B, Seligman J, Tadir Y, Ovadia J, Shalgi R. Thiol status in human sperm. Molec Reprod Develop 1991; $\omega$ 29:282-8.

38 Oba T, Yamaguchi M, Wang S, Johnson JD. Modulation $\stackrel{S}{=}$ of the $\mathrm{Ca}^{2+}$ channel voltage sensor and excitation-concentration coupling by silver. Biophys $\mathcal{f} 1992 ; 63: 1416-20$. 